\title{
Theoretical and experimental studies of dielectric two-dimensional Bragg structures for development of spatially-extended heterolasers
}

\author{
N.Yu. Peskov, V.R. Baryshev, N.S. Ginzburg, E.R. Kocharovskaya, A.M. Malkin, \\ D.M. Padozhnikov, M.D. Proyavin and V.Yu. Zaslavsky
}

Institute of Applied Physics RAS, Nizhny Novgorod, Russia, peskov@appl.sci-nnov.ru

\section{Introduction}

Two-dimensional distributed feedback (2D DF) was originally proposed [1] for obtaining super-power coherent radiation in relativistic masers based on spatially-extended electron beams. In this case, the 2D $\mathrm{DF}$ mechanism is realized in a $2 \mathrm{D}$-periodic metallic Bragg structures with doubly periodic corrugation, which due to the arising in them of the transverse wave-flaxes allow synchronization of radiation of wide electron beams and the establishment of a single-mode oscillation regime when their transverse dimensions are in orders of magnitude greater than the radiation wavelength. To date, the operability of the new feedback mechanism has been experimentally demonstrated in the FEMs, which were elaborated in the millimeter wavelength range (from $\mathrm{Ka}$ - up to $\mathrm{W}$ bands) under a record transverse size of the interaction space, reaching up to 50 wavelengths, and the output power level of $\sim 50-100 \mathrm{MW}[2,3]$.

At the same time, high potential of the new feedback mechanism is not exhausted by microwave relativistic generators. In this aspect it should be noted that currently a 1D feedback mechanism, which is realized in "traditional" single-periodical Bragg structures based on the coupling and mutual scattering of two counter-propagating waves, is widely used in quantum DF lasers [4,5]. However, the transverse sizes of such generators in the conditions of maintaining single-mode narrow-band oscillation are limited by several wavelengths, and further enhance of dimensions leads to a complication of the radiation spectrum and, thus, loss of its spatial coherence. One of the attractive ways to solve this problem in heterolasers is the use of novel 2D feedback mechanism (Fig. 1), which in this case can be realized in 2D Bragg structures of planar geometry with doubleperiodical modulation of the effective refractive index

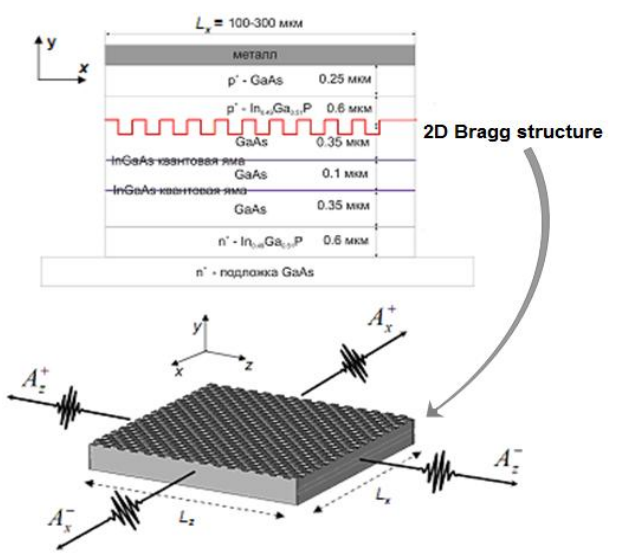

Fig. 1. Scheme of heterolaser based on 2D Bragg structure. of the dielectric waveguide. According to the theoretical analysis carried out in [6], the use of these structures makes it possible to synchronize the radiation from the active medium characterized by large Fresnel parameters in both transverse directions.

The paper presents results of theoretical analysis and computer simulations of the electrodynamic properties of dielectric 2D Bragg structures of planar geometry. "Cold" tests of prototypes of the structures were carried out in the W-band and demonstrated good coincidence with the design parameters. Possibility to advance structures of this type in the sub-mm ranges is discussed.

\section{Results of simulations and "cold" tests in W-band}

One of the main issues of the study was the analysis of acceptable technologies for the manufacture of planar dielectric Bragg structures. For operation at $\mathrm{mm}$ wavelengths, the technology based on the 3D computer printing, which characterized by a high speed of production and affordable cost of the models, was developed.

An important aspect for the discussed technology is the study of dielectric properties of plastics used for printing (which are practically not available in the standard descriptions and strongly depend on the quality and printing characteristics). Complex dielectric permittivities of different plastics were tested using a network analyzer in the Ka- and W-bands by measuring the reflection and transmission coefficients through a standard waveguide having the inserts from these dielectrics. The values of dielectric permittivity (complex and imaginary parts) were found by comparing the measured characteristics with the calculated ones (using iterative procedure). As a result of the experiments were selected plastics, the most suitable for the manufacture of the structures. Measured value of the dielectric constant was $\operatorname{Re} \varepsilon=2.4$ and $\tan \delta=0.0015$. At the same time, the experiments did not identify strong frequency dependences of these coefficients in tested bands.

Simulations of planar dielectric 2D Bragg structures were performed using the commercial code CST "Microwave Studio". Parameters for the simulations were chosen similar to the conditions of the experiments described below. Results of simulations of the frequency dependences of the integral scattering coefficients on the 2D structure (reflection, transmission, and transverse scattering coefficients) are presented in Fig. 2. It is showed good agreement with the results of previous theoretical analysis carried out in [6]. In the designed region of parameters the effective Bragg 
scattering zone corresponding to the coupling of the four partial wave-flaxes, which possess the structure of the TM-wave of the dielectric waveguide propagating over the corrugated surface, was verified.

Experimental study of 2D Bragg dielectric structures was conducted in the W-band. The thickness of the dielectric waveguide was $2 \mathrm{~mm}$, the so-called "chessboard" corrugation was made on this waveguide with a period along each $(x$ and $z$ ) directions $d_{\mathrm{x}}=d_{\mathrm{z}}=4 \mathrm{~mm}$ and a depth of $0.5 \mathrm{~mm}$ (Fig. 3). The transverse dimensions of the structure were $18 \mathrm{~cm} \times 18 \mathrm{~cm}$, the structure (dielectric waveguide) was placed on a substrate of duralumin.

A network analyzer P2-67 was used as the RF signal source. To form incident wave-beam (and receiving scattered radiation), the planar quasi-optical transmission line, which provides at its output a wide wave-beam with the structure of TEM-wave and plane phase, was exploited (Fig. 3). The results of "cold" measurements of the reflection and transmission coefficients on the structure described above are shown in Fig. 4. In accordance with the calculations, an effective Bragg scattering zone was observed in the vicinity of $60 \mathrm{GHz}$. Comparison Fig. 2 and 4 demonstrates good agreement between the simulations and "cold" tests results both on the position and width of the Bragg zone as well as on the amplitude of the integral scattering coefficients.

\section{Summary and discussions}

Thus, theoretical and experimental studies have confirmed the feasibility and operability of the 2D dielectric Bragg structures in the $\mathrm{mm}$ wavelength band. The available technology of manufacturing structures of this type using the 3D printing was developed. The good coincidence of the electrodynamic properties of the 2D structures measured in "cold" tests with the results of computer simulations based on commercial code CST "Microwave Studio" was demonstrated. The experimental studies have confirmed that the dielectric properties of plastics utilized

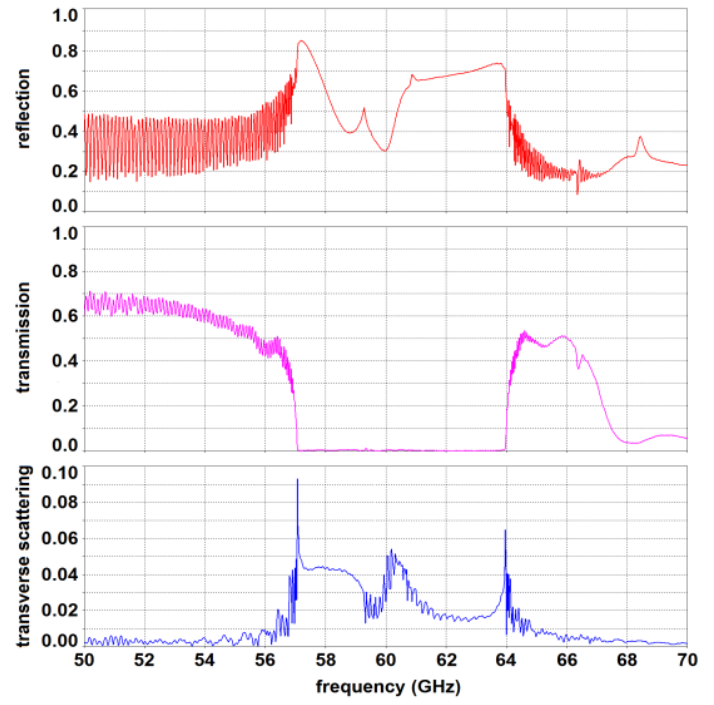

Fig. 2. Results of CST - simulations of dielectric 2D Bragg structure having parameters close to experimental prototype. Frequency dependencies of the reflection (top), transmission (middle) and transverse scattering (bottom) coefficients.

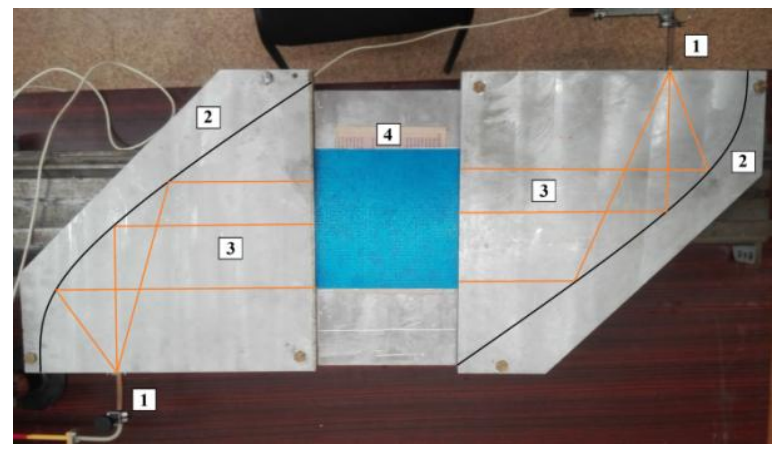

Fig. 3. Scheme of the "cold" tests: 1 - standard single-mode waveguides powered by the network analyzer, 2 - planar quasi-optical transmission lines, 3 - incident and transmitted wave-beams, 4 - dielectric 2D Bragg structure.
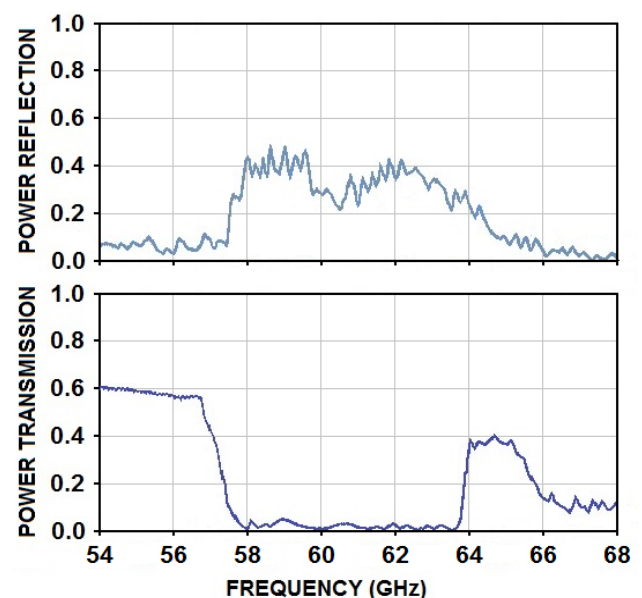

Fig. 4. Results of "cold" measurements of frequency dependencies of the reflection (top) and transmission (bottom) coefficients of dielectric 2D Bragg structure in the W-band.

in the proposed technology are adequate for manufacturing models of dielectric 2D structures with the accuracy acceptable for operation up to a short-wave part of the mm wavelength band. The further development of the 2D Bragg structures of the discussed type should be directed to advance into the sub-mm range when using the industrial $3 \mathrm{D}$ printers and the polymer photo printing technology, the claimed accuracy of which currently reaches up to $1 \mu \mathrm{m}$.

This work is partially supported by the Russian Foundation for Basic Research (grant 18-48-520022).

\section{References}

1. N.S.Ginzburg, N.Yu.Peskov, A.S.Sergeev. Dynamics of free-electron lasers with two-dimension distributed feedback // Optics Commun. 1994. V.112. P.151.

2. N.S.Ginzburg, N.Yu.Peskov, A.S.Sergeev, e.a. Production of powerful spatially coherent radiation in planar and coaxial FEM exploiting two-dimensional distributed feedback // IEEE Trans. on Plasma Sci. 2009. V.37. No.9. P.1792.

3. A.V.Arzhannikov, N.S.Ginzburg, P.V.Kalinin, e.a. Using two-dimensional distributed feedback for synchronization of radiation from two parallel-sheet electron beams in a FreeElectron Maser // Phys. Rev. Lett. 2016. V.117. P.114801.

4. H.Kogelnik, C.V.Shank. Stimulated emission in a periodic structure // Appl. Phys. Lett. 1971. V.18, No.4. P.152.

5. A.Yariv. Quantum Electronics // John Wiley and Sons Inc., New York, 1975.

6. N.S.Ginzburg, V.R.Baryshev, A.S.Sergeev, A.M.Malkin. Dynamics of semiconductor lasers with two-dimensional distributed feedback // Phys. Rev. A. 2015. V.91. P.053806. 